\title{
Ancillary Ultrasonic Rangefinder for Autonomous Vehicles
}

\author{
Yair Wiseman \\ Computer Science Department, Bar-Ilan University \\ Ramat-Gan 52900, Israel \\ wiseman@cs.biu.ac.il
}

\begin{abstract}
The latest fatal accidents of autonomous vehicle happened because of undetected peer vehicles or persons. These fatal accidents call attention to the rangefinder devices which are a very significant component of every autonomous vehicle. The rangefinder is the starting point for the decision whether the vehicle should continue to go, to stop, or bypassing an object. In this paper an ultrasonic based rangefinder device for autonomous vehicles is presented. The device can be used as an ancillary system in addition to standard LIDAR system used by almost all the autonomous vehicles, with the aim of overcoming the obstructions of conventional LIDAR systems.
\end{abstract}

Keywords: Rangefinder, autonomous vehicles, Ultrasonic

\section{Introduction}

Using an ultrasonic device for range finding in an autonomous vehicle [1] has been suggested in the part years [2]. The method has been also prevalently implemented in regular vehicles for parking sensors [3]. There are also security implementations for this technology [4].

LIDAR range sensors [5] have a critical obstruction. A LIDAR beam is narrow [6]. This quality can be an advantage for some aspects; however, a narrow beam is also a significant disadvantage because the beam will be prone to disregard narrow objects like for example lampposts; therefore, the autonomous vehicle will have to take a lot of readings when scanning its surrounding using a LIDAR based rangefinder, because the more readings per revolution, the better the chance of picking up narrow objects [7]; hence ultrasonic devices for rang finding in autonomous vehicles are suggested at least for a backup for the LIDAR rangefinder.

\section{Ultrasonic Rangefinder}

The concept of ultrasonic devices is to emit acoustic pulses. After that, the device measuring the time it took for the reflected signal to be detected. The speed of sound is a known number, but it is affected by temperature, humidity and air pressure [8]; however, typically the speed of sound is $700 \mathrm{MPH}$ to $800 \mathrm{MPH}$. The distance is time multiplied by speed so accordingly the device can calculate distances of objects in its surroundings. The inaccuracy of the result because of the differences in the speeds of sound is ignored, because autonomous vehicles anyway take a safety factor when taking their decisions [9].

The range of common parking sensors is usually about 10 feet [10] because there is no need in parking sensors to detect farer objects [11,12]. There are also some parking sensors with long-lasting signals even for 70 feet [13]; however, in a range finder for autonomous vehicles, it is necessary to detect much farer objects.

Received (June 14, 2018), Review Result (August 5, 2018), Accepted (August 19, 2018) 
Particularly, in a high-speed highway, the vehicle will not be able to come to a full stop in 10 feet, so there is a necessity to send a stronger signal, so the signal can be detected even after a long distance.

The safe stopping distance is the sum of the reaction distance and the braking distance [14]. The average reaction time of a regular driver is approximately $3 / 4$ seconds [15]; however, the reaction time of an autonomous vehicle is almost zero and actually this time is neglected in the braking time and accordingly the reaction distance is also neglected.

Braking distance [16] i.e., the distance that the vehicle goes from the start of the braking system operation until the vehicle will come to a full stop depends on several various factors:

- The speed of the vehicle [17].

- The weather [18].

- The condition of the vehicle and its tires and brakes [19, 20,21].

- The road condition [22].

- The intensity of the press on the brake pedal by the driver [23].

It should be also noted that the braking distance is not increased in linear to the increase in speed, but increased in square to the increase in speed. For example, if the speed of a certain vehicle increases twofold, the braking distance will be four times longer.

Vehicle's velocity can significantly vary from a vehicle creeping forward in a traffic jam $[24,25,26]$ or a vehicle goes in an empty highway. The distance for a vehicle goes in $75 \mathrm{MPH}$ to come to a full stop is approximately $250 \mathrm{feet}$; hence our system was designed to send strong enough signals that can last for more than 300 feet.

\section{Implementation}

The ultrasonic rangefinder consists of a control unit, an ultrasonic transmitter, an ultrasonic receiver and an asynchronous counter. They are connected as is depicted in Figure 1.

Like the implementation of many other Intelligent Transportation Systems $[27,28]$, The control unit of the rangefinder device has been designed as a Moore state machine [29]. The control unit has just 3 states. The output lines of the state machine go to the counter and to the ultrasonic transmitter.

In the first state the control unit instructs the counter to start the counting. In addition, in this state the control unit instructs the ultrasonic transmitter to emit its signal.

Next, the state machine goes into a wait state. The state machine waits for a return signal from a possible object.

If a signal is detected, the machine goes to another state where the machine outputs the value of the counter. 


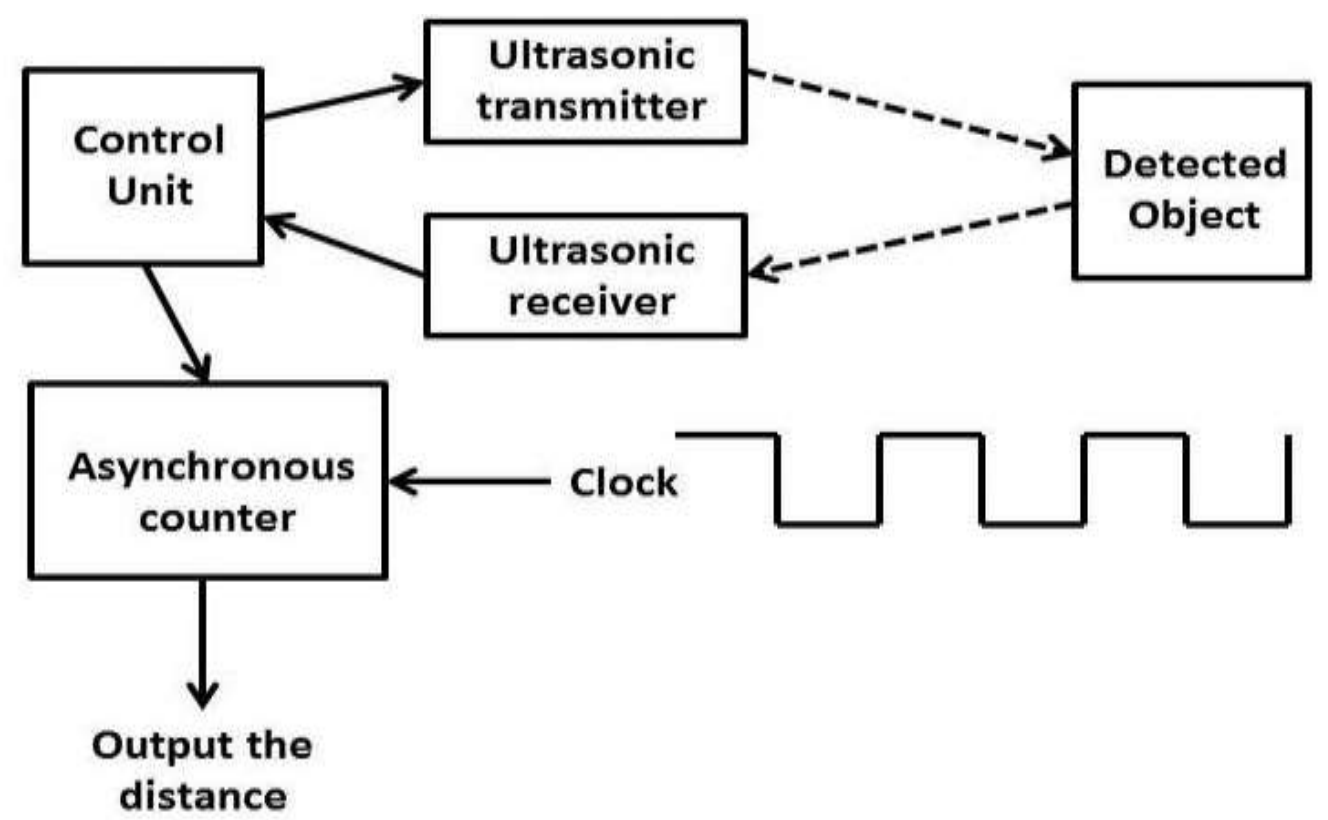

Figure 1. The Ultrasonic Rangefinder

If the counter returns to zero, the machine will understand that no object has been detected and the therefore the machine will return to the first step, where the machine instructs the counter to start counting and instructs the ultrasonic transmitter to emit its signal.

A description of the state machine is shown in Figure 2.

Given that the control unit has 3 states, it contains 2 flip-flops according to the formula:

Number of flip-flops $=\left\lceil\log _{2}(\right.$ number of states $\left.)\right\rceil$

Let us denote the flip-flops as FF1, FF0. We also denote the input from the ultrasonic receiver as US and the overflow of the counter (i.e. when the counter passes the value of 511) as CO.

The next state functions of the control unit's flip-flops are described in Karnaugh maps [30] in Figure 3 and Figure 4. Accordingly, the next state functions for both of the flip-flops are:

$$
\begin{aligned}
& \text { Next FF1 }=F F 0 \cdot U S \cdot \overline{C O} \\
& \text { Next FF0 }=\overline{F F 1} \cdot \overline{F F 0}+F F 0 \cdot \overline{U S} \cdot \overline{C O}
\end{aligned}
$$




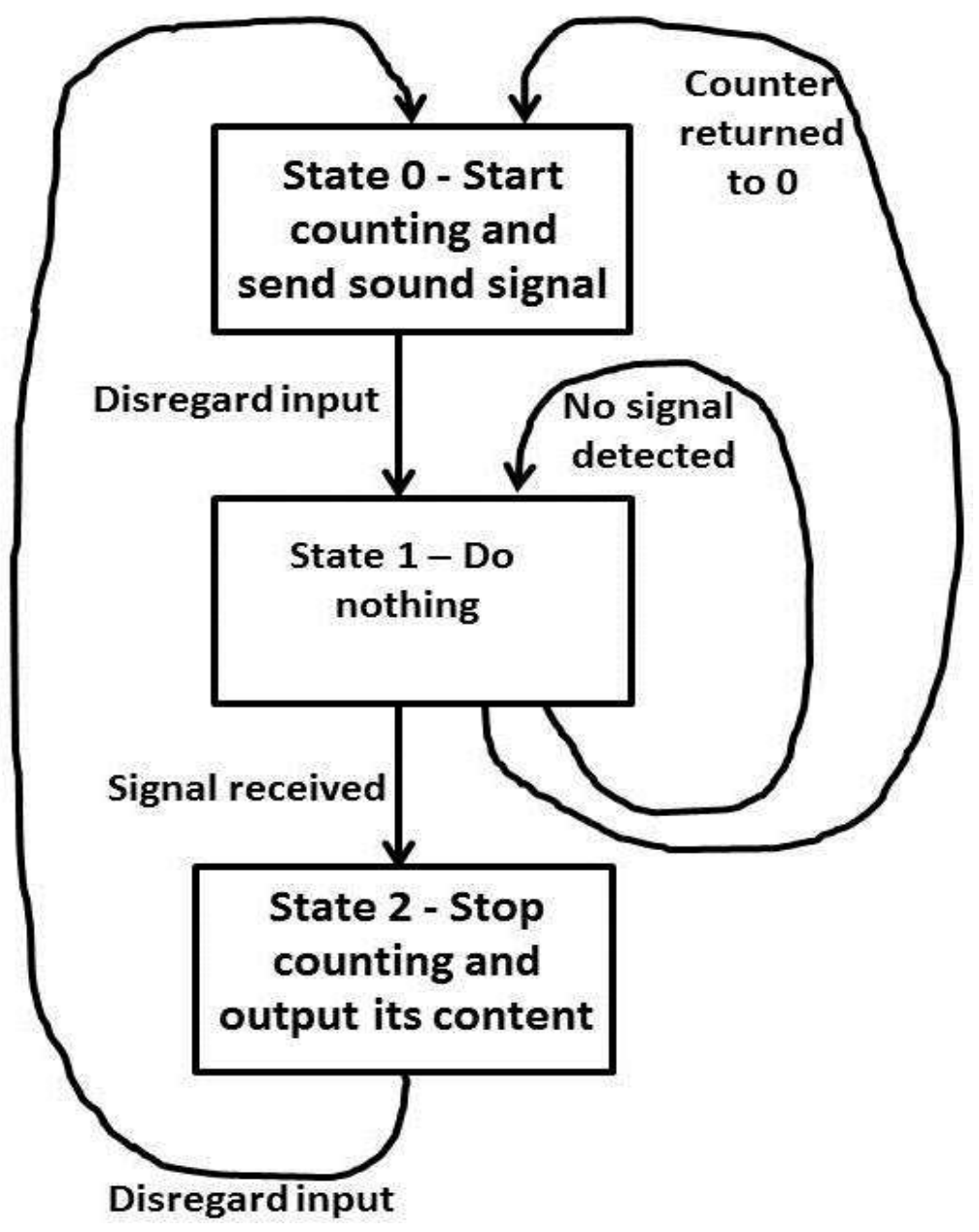

Figure 2. Diagram of the State Machine

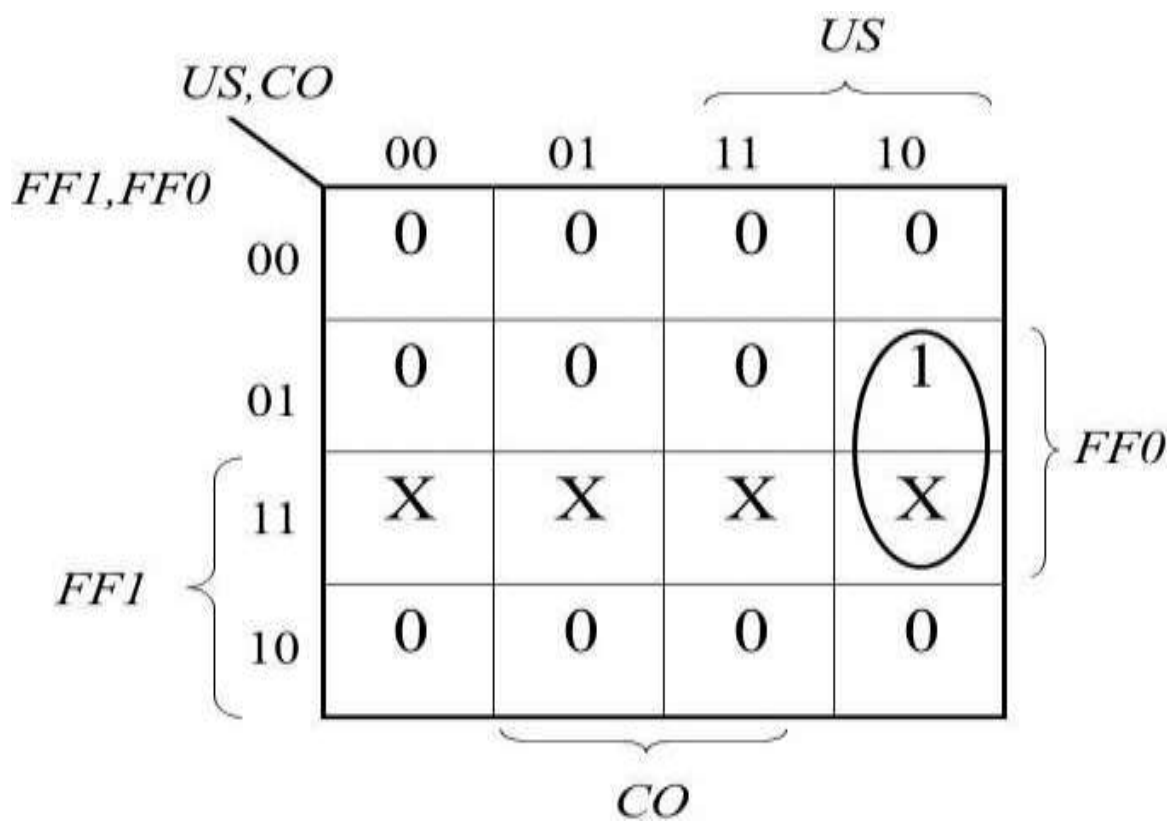

Figure 3. Karnaugh Map for Next State of FF1 


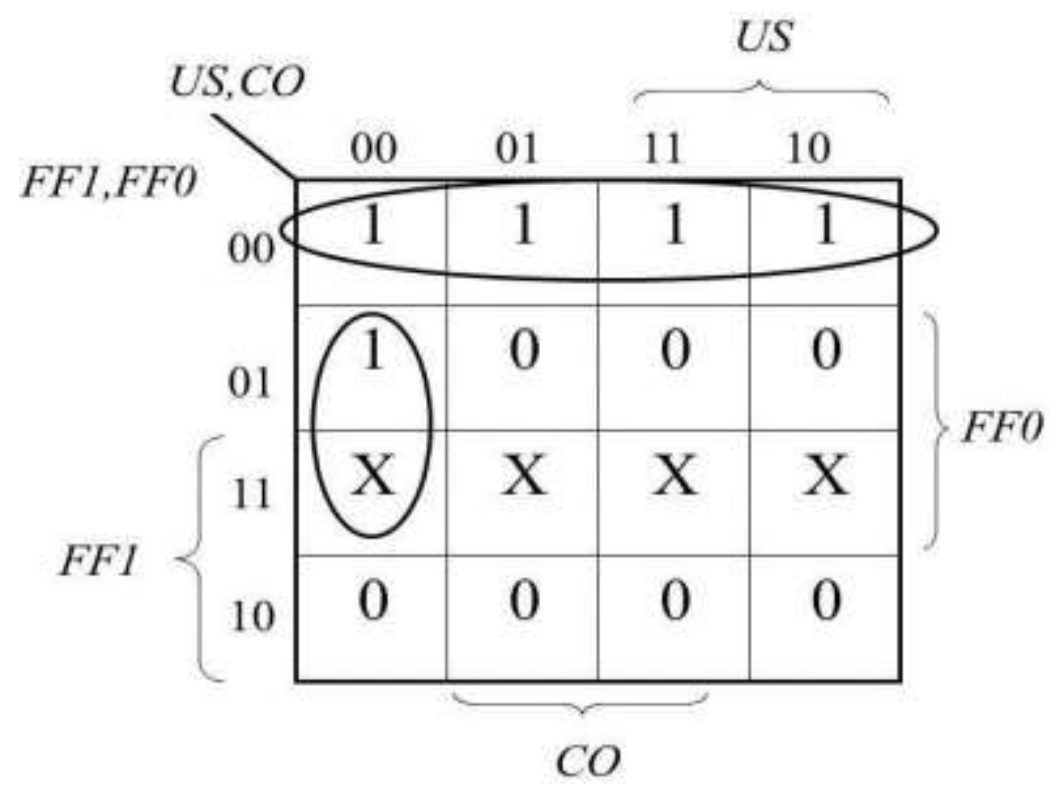

Figure 4. Karnaugh Map for Next State of FF1

Consequently, the circuits of the next values of flip-flops are shown in Figure 5 (Next FF1) and Figure 6 (Next FF0).

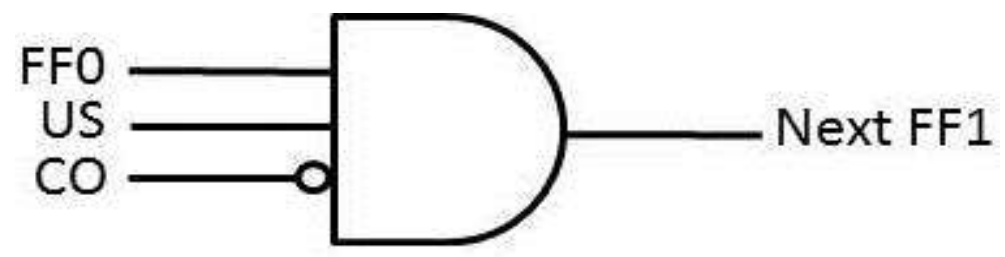

Figure 5. Next FF1 Circuit

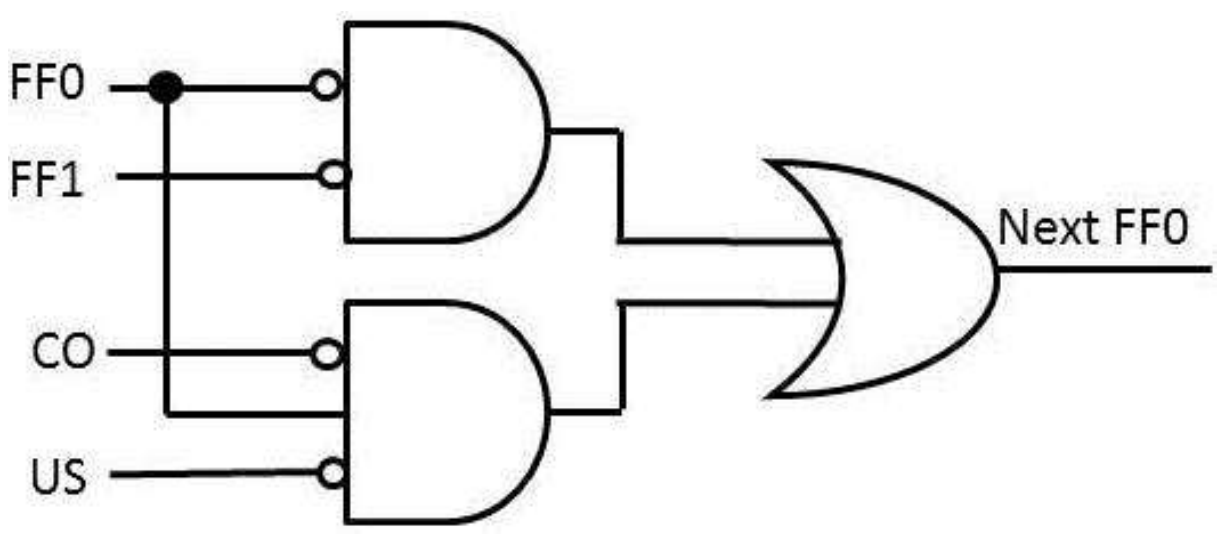

Figure 6. Next FF0 Circuit

\section{The Rangefinder Counter}

An asynchronous counter consists of JK flip-flops or T flip-flops whose inputs are connected to a voltage that represent the logical value of "1" [31]. The "1" input causes the current output of the flip-flops to be the inverse of its previous output. Each flip-flop stores one bit, so it can count from 0 to $2^{\mathrm{n}}-1$ where $\mathrm{n}$ is the number of 
the flip-flops in the counter, before the counter's value returns to 0 . Actually an overflow occurs, but it is ignored.

The number stored in the first flip-flop will change in each clock cycle; therefore, it will take two clock cycles until the first flip-flop returns to its original value. In each clock cycle the flip-flop's value will change from 0 to 1 or from 1 to 0 , so as a result, the output of the first flip-flop creates a new clock whose length is the same as the two cycles of the original clock, that is, the frequency of this new clock is half the frequency of the original clock. This new clock is connected as a clock signal to a second flip-flop. The second flip-flop will be, like the first flip-flop, a one-bit counter, but its pace will be half the speed of the first unit. The output of the second flip-flop is connected as a clock to a third flip-flip that its pace will be half the speed of the second flip-flop.
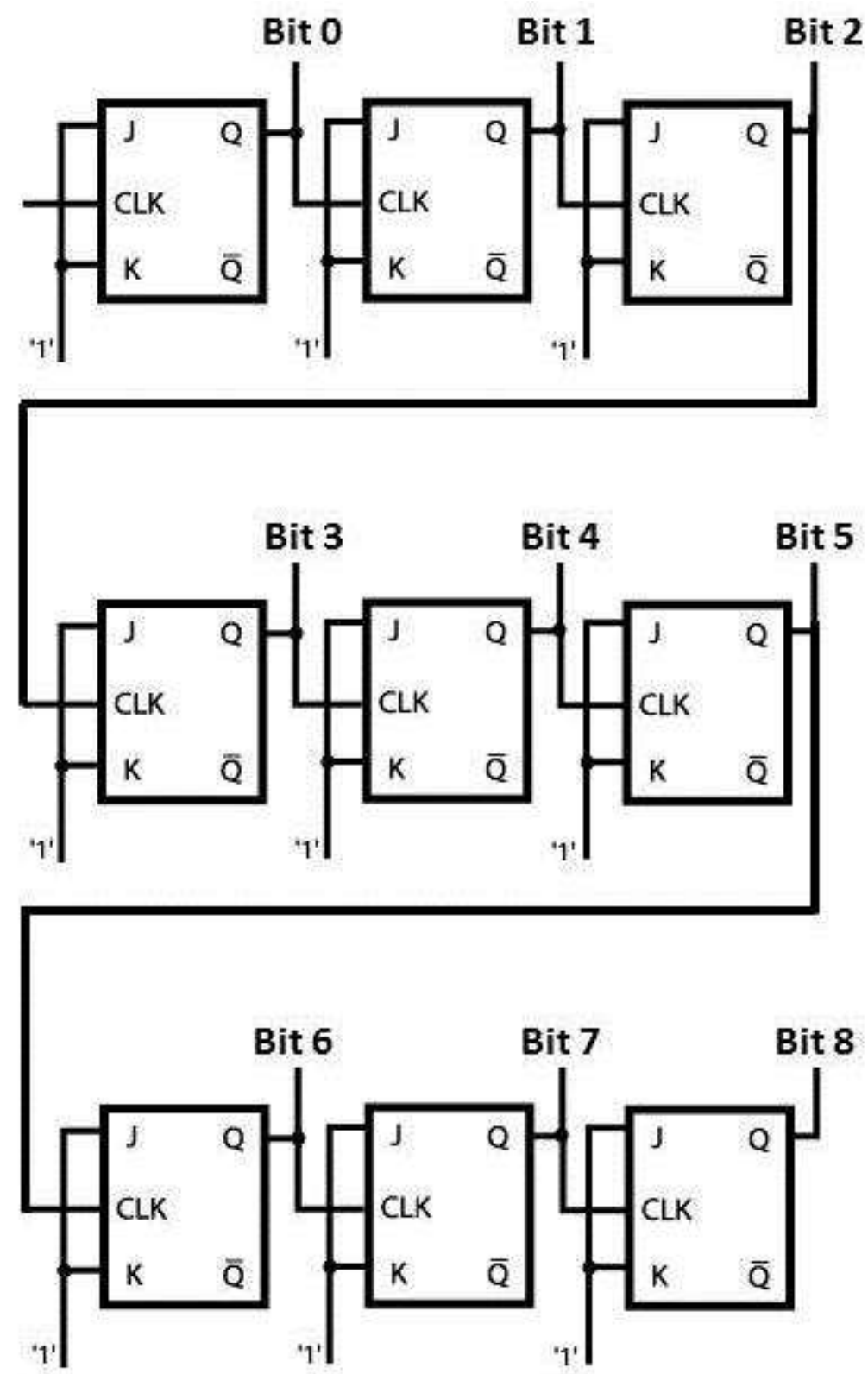

Figure 7. Nine Bits Asynchronous Counter 
A combination of several flip-flops together will generate a several bit counter, as can be seen in Figure 7. Particularly, the counter in Figure 7 consists of nine flipflops where Bit 0 is the first flip-flop which inverses the fastest and Bit 8 is the last flip-flop which inverses the slowest.

The use of the flip-flops output as a clock causes time discrepancies between the bits of the counter, because when the counter value is modified, the flip-flop that stores Bit 0 is modified first and it takes time until a possible modification is propagated to the rest of the flip-flops [32].

As a result, such a counter is not suitable for use in regular synchronous circles where all components are modified at the same time by a single clock signal and a short modification time is imperative. A modification that propagates in linear time to the number of the flip-flops can be too slow for several implementations; however, in the implementation of this paper, anyway the clock cycle is quite long, so such a long modification time will not be a problem.

We made use of a maximum range of 300 feet which the sound should go twice (back and forth). In the speed of sound going 300 feet will take about half a second. We designed a counter of 9 bits which represents a resolution of 512 options. This led us to adjust the clock of the counter to be $1 \mathrm{KHZ}$, which will be able to count to 0.511 seconds.

\section{The Clock}

The clock we used is actually a kind of an electronic oscillator [33]. This electronic circuit produces a periodic, oscillating electronic signal as a square wave by converting direct current (DC) from a power supply to a square wave signal.

The electronic oscillator is described in Figure 8. Let us suppose Capacitor L $_{\mathrm{L}}$ is discharged whereas Capacitor ${ }_{R}$ is charged. In that case, an electric current will flow through Capacitor ${ }_{L}$ which will continue to the base of Transistor $_{R}$. This electric current will continue to the $0 \mathrm{~V}$ through the emitter of Transistor $_{\mathrm{R}}$. This electric current will make Transistor $_{\mathrm{R}}$ being almost a zero resistance; hence the output voltage will be almost $0 \mathrm{~V}$.

While this happens, Capacitor ${ }_{R}$ discharges its voltage through Transistor $_{R}$ and Capacitor $r_{L}$ is charged by the electric current flows through it, so the Capacitors switch roles. The electric current flows through Capacitor $_{R}$, while Capacitor $_{L}$ discharges its voltage through Transistor $_{\mathrm{L}}$.

The resistors $\mathrm{R}_{\mathrm{L}}$ is very important when Transistor $_{\mathrm{L}}$ has a significant electric current flow from the base to the emitter and it actually becomes almost a zero resistance. In such a case a short circuit will be formed if resistor $R_{L}$ was not placed there. Similarly, $R_{R}$ eliminates a short circuit in a case where a significant electric current flow from the base to the emitter of Transistor ${ }_{R}$.

This constant switching roles cause the voltage output to be as described in the bottom of Figure 8. 


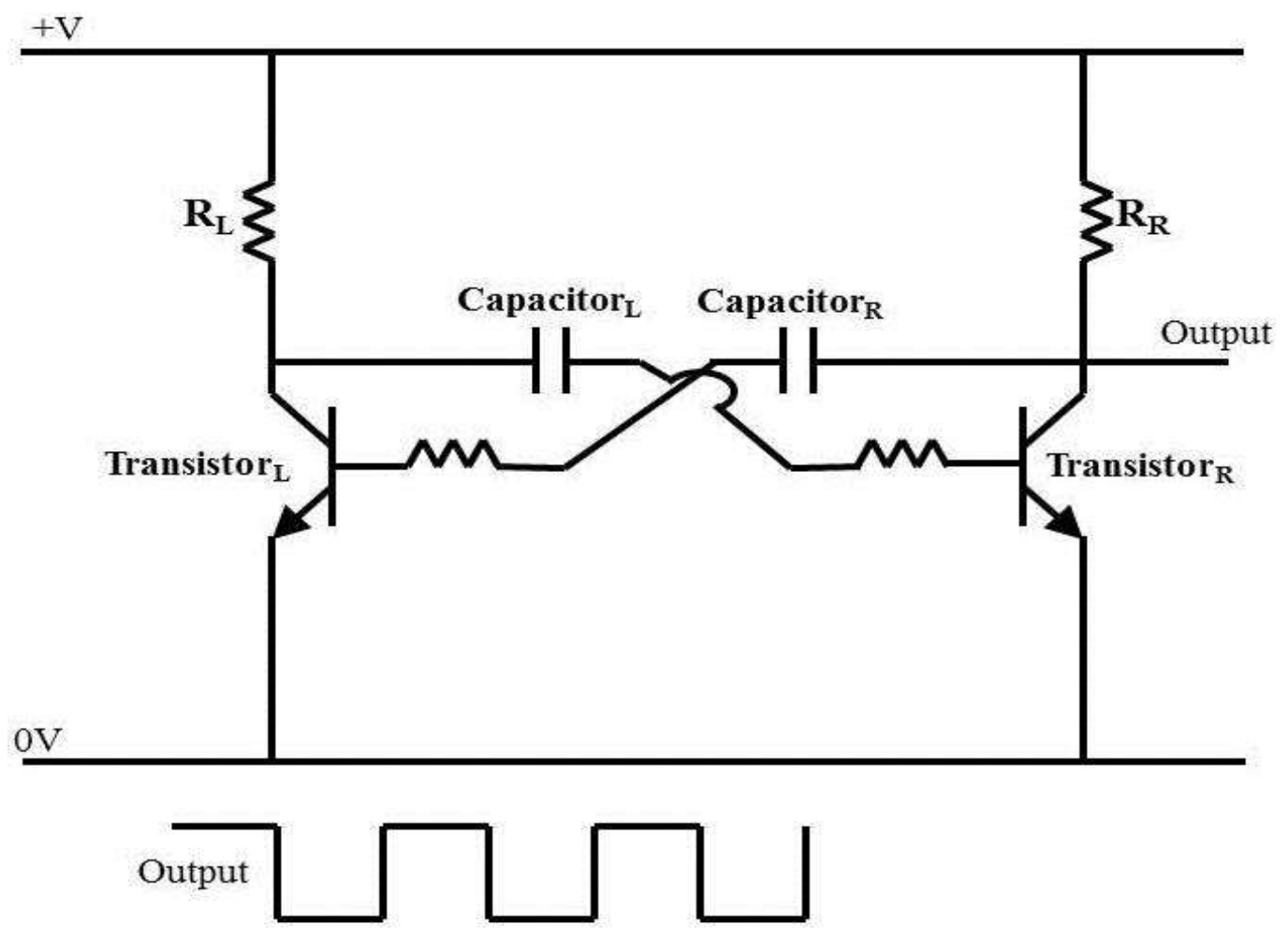

Figure 8. Square Wave Clock

\section{Conclusions}

The suggested incorporated system of a LIDAR rangefinder and an ultrasonic ranger finder can be implemented in autonomous vehicles with a significantly better ability of object detection, for the reason that the suggested device can help in overcoming the obstructions of conventional LIDAR systems.

This better ability is an important advantage in avoiding vehicle accidents [34, $35,36,37]$, because undetected objects or wrong range estimation may possibly lead to a collision and a fatal accident might occur $[38,39]$, so the rangefinder should be as faultless as possible so as to save lives.

\section{References}

[1] Y. Wiseman, “Autonomous Vehicles", Encyclopedia of Organizational Knowledge, Administration, and Technologies, Available online at: http://u.cs.biu.ac.il/ wiseman/autonomousr.pdf, (2019).

[2] F. Figueroa and A. Mahajan, "A robust navigation system for autonomous vehicles using ultrasonics", Control Engineering Practice, vol. 2, no. 1, (1994), pp. 49-59.

[3] W. J. Park, B. S. Kim, D. E. Seo, D. S. Kim and K. H Lee, "Parking space detection using ultrasonic sensor in parking assistance system", In Proceedings of IEEE Intelligent Vehicles Symposium, (2008), pp. 1039-1044.

[4] Y. Wiseman and Y. Giat, "Multi-modal passenger security in Israel", Multimodal Security in Passenger and Freight Transportation: Frameworks and Policy Applications, Edward Elgar Publishing Limited, Chapter 16, (2016), pp. 246-260.

[5] A. G. Kashani, M. J. Olsen, C. E. Parrish and N. Wilson. "A review of LiDAR radiometric processing: From ad hoc intensity correction to rigorous radiometric calibration", Sensors, vol. 15, no. 11, (2015), pp. 28099-28128.

[6] A. T. Pedersen and M. Courtney, "Flywheel Calibration of Coherent Doppler Wind Lidar", In proceedings of: European Lidar Conference, (2018).

[7] J. Petit, B. Stottelaar, M. Feiri and F. Kargl, "Remote attacks on automated vehicles sensors: Experiments on camera and lidar”, Black Hat Europe, vol. 11, (2015), pp. 1-13. 
[8] G. S. K. Wong and T. F. W. Embleton, "Variation of the Speed of Sound in Air with Humidity and Temperature", The Journal of the Acoustical Society of America, published by American Institute of Physics (AIP), vol. 77, no. 5, (1985), pp. 1710-1712.

[9] P. Hosur, R. B. Shettar and M. Potdar, "Environmental awareness around vehicle using ultrasonic sensors", In 2016 IEEE International Conference on Advances in Computing, Communications and Informatics (ICACCI), (2016), pp. 1154-1159.

[10] K. Hamada, Z. Hu, M. Fan and H. Chen, "Surround View Based Parking Lot Detection And Tracking", In proceedings of 2015 IEEE Intelligent Vehicles Symposium (IV), Seoul, South Korea, (2015), pp. 1106-1111.

[11] Y. Wiseman, "Remote Parking for Autonomous Vehicles", International Journal of Hybrid Information Technology, vol. 10, no. 1, (2017), pp. 313-324.

[12] Y. Wiseman, "Self-Driving Car - A Computer will Park for You", International Journal of Engineering \& Technology for Automobile Security, SERSC, Sandy Bay, Australia, vol. 1, no. 1, (2017), pp. 9-16.

[13] D. S. Vidhya, D. P. Rebelo, C. J. D’Silva, L. W. Fernandes and C. J. Costa, "Adaptive Cruise Control", International Journal for Innovative Research in Science \& Technology, vol. 3, no. 1, (2016), pp. 142146.

[14] D. Fambro, R. Koppa, D. Picha and K. Fitzpatrick, "Driver perception-brake response in stopping sight distance situations", Transportation Research Record: Journal of the Transportation Research Board, vol. 1628, (1998), pp. 1-7.

[15] T. Nguyen, R. Hau and J. Bartlett, "Driving Reaction Time Before and After Anterior Cruciate Ligament Reconstruction", Knee Surgery, Sports Traumatology, Arthroscopy, vol. 8, no. 4, (2000), pp. 226-230.

[16] E. Coelingh, A. Eidehall and M. Bengtsson, "Collision Warning with Full Auto Brake and Pedestrian Detection-A Practical Example of Automatic Emergency Braking”, In proceedings of 2010 13th International IEEE Conference on Intelligent Transportation Systems (ITSC), (2010), pp. 155-160.

[17] J. C. Gerdes and J. K. Hedrick, "Vehicle speed and spacing control via coordinated throttle and brake actuation", Control Engineering Practice, Published by Elsevier, vol. 5, no. 11, (1997), pp. 1607-1614.

[18] A. Khattak, P. Kantor and F. Council, "Role of adverse weather in key crash types on limited-access: roadways implications for advanced weather systems", Transportation Research Record, vol. 1621, (1998), pp. 10-19.

[19] Y. Wiseman, "Take a Picture of Your Tire"!, In Proceedings of The $12^{\text {th }}$ IEEE Conference on Vehicular Electronics and Safety (IEEE ICVES-2010), Qingdao, ShanDong, China, (2010), pp. 151-156.

[20] Y. Wiseman, "The Effectiveness of JPEG Images Produced by a Standard Digital Camera to Detect Damaged Tyres", World Review of Intermodal Transportation Research, vol. 4, no. 1, (2013), pp. 23-36.

[21] Y. Wiseman, "Camera That Takes Pictures of Aircraft and Ground Vehicle Tires Can Save Lives", Journal of Electronic Imaging, vol. 22, no. 4, 041104, (2013).

[22] W. F. Rogge, L. M. Hildemann, M. A. Mazurek, G. R. Cass and B. R.T. Simoneit, "Sources of Fine Organic Aerosol. 3. Road Dust, Tire Debris, and Organometallic Brake Lining Dust: Roads as Sources and Sinks", Environmental Science and Technology, ACS Publications, vol. 27, no. 9, (1993), pp. 18921904.

[23] L. Evans and P. H. Gerrish, "Antilock brakes and risk of front and rear impact in two-vehicle crashes", Accident Analysis and Prevention, Published by Elsevier, vol. 28, no. 3, (1996), pp. 315-323.

[24] Y. Wiseman, "Real-Time Monitoring of Traffic Congestions", The $17^{\text {th }}$ IEEE International Conference on Electro Information Technology (EIT 2017), Lincoln, Nebraska, USA, (2017), pp. 501-505.

[25] Y. Wiseman, "Computerized Traffic Congestion Detection System", International Journal of Transportation and Logistics Management, vol. 1, no. 1, (2017), pp. 1-8.

[26] Y. Wiseman, "Tool for Online Observing of Traffic Congestions", International Journal of Control and Automation, vol. 10, no. 6, (2017), pp. 27-34

[27] Y. Wiseman, "Conceptual Design of Intelligent Traffic Light Controller", International Journal of Control and Automation, vol. 9, no. 7, (2016), pp. 251-262.

[28] Y. Wiseman, "Traffic Light with Inductive Detector Loops and Diverse Time Periods", Contemporary Research Trend of IT Convergence Technology, SERSC Australia, vol. 4, (2016), pp. 166-170.

[29] M. Morrison and N. Ranganathan, "Design of a Moore Finite State Machine Using a Novel Reversible Logic Gate, Decoder and Synchronous Up-Counter", In proceedings of 2011 11th IEEE Conference on Nanotechnology (IEEE-NANO-2011), Portland, Oregon, USA, (2011) August 15-18, pp. 1445-1449.

[30] M. E. Holder, "A modified Karnaugh map technique", IEEE Transactions on Education, vol. 48, no. 1, (2005), pp. 206-207.

[31] M. M. Mano and C. R. Kime, "Logic and computer design fundamentals", Pearson Prentice Hall, Upper Saddle River, NJ, (2008).

[32] L. A. Lim, A. Ghazali, S. Chan, T. Yan and C. C. Fat, "Sequential circuit design using quantum-dot cellular automata (qca)", In proceedings of 2012 IEEE international conference on Circuits and systems (ICCAS), (2012), pp. 162-167.

[33] W. S. Chung, H. Kim, H. W. Cha and H. J. Kim, "Triangular/square-wave generator with independently controllable frequency and amplitude", IEEE transactions on instrumentation and measurement, vol. 54, no. 1, (2005), pp. 105-109. 
[34] Y. Wiseman and I. Grinberg, "The Trolley Problem Version of Autonomous Vehicles", The Open Transportation Journal, vol. 12, (2018), pp. 105-113.

[35] Y. Wiseman and I. Grinberg, "Autonomous Vehicles Should Not Collide Carelessly", Advanced Science and Technology Letters, vol. 133, (2016), pp. 223-228.

[36] Y. Wiseman and I. Grinberg, "When an Inescapable Accident of Autonomous Vehicles is Looming", International Journal of Control and Automation, SERSC Australia, vol. 9, no. 6, pp. 297-308, (2016).

[37] Y. Wiseman and I. Grinberg, "Circumspectly Crash of Autonomous Vehicles", Proc. IEEE International Conference on Electro Information Technology (EIT 2016), Grand Forks, North Dakota, USA, (2016), pp. 382-386.

[38] I. Grinberg and Y. Wiseman, "Scalable Parallel Collision Detection Simulation", Proc. Signal and Image Processing (SIP-2007), Honolulu, Hawaii, (2007), pp. 380-385.

[39] I. Grinberg and Y. Wiseman, "Scalable Parallel Simulator for Vehicular Collision Detection", International Journal of Vehicle Systems Modelling and Testing, Inderscience, vol. 8, no. 2, (2013), pp. $119-144$

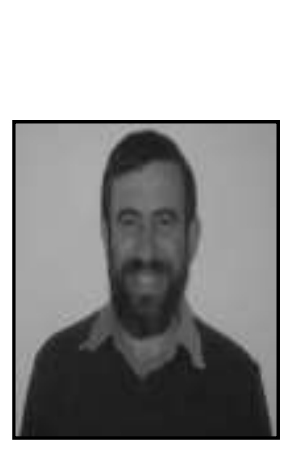

\section{Author}

Yair Wiseman, received the M.Sc. (summa cum laude) and Ph.D. degrees from Bar-Ilan University, the first Post-Doctoral degree with the Hebrew University of Jerusalem, and the second Post-Doctoral degree with the Georgia Institute of Technology.

His research interests include autonomous vehicles, intelligent transportation systems, embedded systems, real-time systems, computational transportation science, operating systems, and process scheduling.

Dr. Wiseman has authored two books. He is on the editorial board of several journals, a member of dozens of conference committees and a reviewer of many scholarly journals.

Dr. Wiseman has been teaching in many institutes including BarIlan University, The Hebrew University of Jerusalem and Israel Aircraft Industry and has been supervising many graduate students. An interesting point is that Albert Einstein is Dr. Wiseman's academic great-great-grandfather (i.e. the advisor of the advisor of the advisor of Dr. Wiseman's advisor).

Dr. Wiseman has collaborated with other partners and received research grants to run an active laboratory from inter alia Sun Microsystems, Intel, and Polak Foundation.

Dr. Wiseman is an international expert who has reviewed and evaluated several large projects of the European Union, Israel Science Foundation, Marie Skłodowska-Curie actions in Ireland, Ministry of Education and Science of Kazakhstan. His papers have been published in many venues around the world. 